\title{
Complete lymphadenectomy following positive sentinel lymph node biopsy in cutaneous melanoma: a critical review*
}

\author{
Daniel Eiger ${ }^{1,2}$, Daniel Arcuschin de Oliveira ${ }^{1,3}$, Renato Leão de Oliveira ${ }^{4}$, Murilo Costa Sousa ${ }^{1,5}$, \\ Mireille Darc Cavalcante Brandão ${ }^{1,6,7,8}$, Renato Santos de Oliveira Filho ${ }^{1,9}$
}

DOI: http:/ / dx.doi.org/10.1590/abd1806-4841.20187312

\begin{abstract}
Cutaneous melanoma is the solid neoplasia with the highest growing incidence among all tumors. It spreads predictably to the lymphatic vessels and sentinel lymph node, and when the latter is affected the prognosis worsens dramatically. Sentinel lymph node biopsy is considered when thickness of the primary tumor exceeds $1 \mathrm{~mm}$ and/or when there are adverse features in thinner melanomas. When there is nodal metastasis, current evidence in the literature recommends complete lymphadenectomy, although this procedure has its intrinsic risks (i.e., lymphedema and cellulitis), and there are no published clinical trials proving additional overall survival benefits. The current in-depth literature review thus aims to identify patients that will benefit most from the procedure, including those with the highest likelihood of presenting additional affected lymph nodes in the same nodal basin. The authors also discuss techniques for identification of the sentinel lymph node, false-negative rates, and predictive models for lymph node involvement. In conclusion, complete elective lymphadenectomy should always be discussed on a case-by-case basis when metastases are detected in the sentinel lymph node.
\end{abstract}

Keywords: Lymph node dissection; Lymphatic metastasis; Melanoma; Sentinel lymph node biopsy

\section{INTRODUCTION}

Cutaneous melanoma (CM) is associated with high lethality. It is currently the malignant neoplasm with the fastest growing incidence of all the tumors, having increased 15-fold in the last 40 years in the United States alone. ${ }^{1}$ Most CMs spread first to the lymphatics, with the sentinel lymph node (SLN) as the first lymph node to receive lymphatic drainage from the skin where the primary lesion is located.

\section{SENTINEL LYMPH NODE BIOPSY}

For cases of localized CM - clinical stages I and II (without clinically detectable lymph nodes), sentinel lymph node (SLN) biopsy is the most accurate staging method. The main variables in the primary lesion for risk of SLN metastasis are Breslow thickness, ulceration, and number of mitoses. SLN biopsy should be considered in all patients with Breslow thickness greater than or equal to $1 \mathrm{~mm}$, as well as for those with thickness less than $1 \mathrm{~mm}$, but greater than $0.75 \mathrm{~mm}$, in the presence of the following adverse factors:

1- Positive deep margins;

2- Lymphatic invasion;

3- Age < 40 years;

4- Significant vertical growth phase;

5- High mitotic index;

6- Clark level IV or greater. ${ }^{2}$

\footnotetext{
Received 17 July 2017.

Accepted 19 January 2018

* Work conducted at Clínica Professor Doutor Renato Santos, São Paulo (SP), Brazil.

Financial support: None.

Conflict of interest: None.

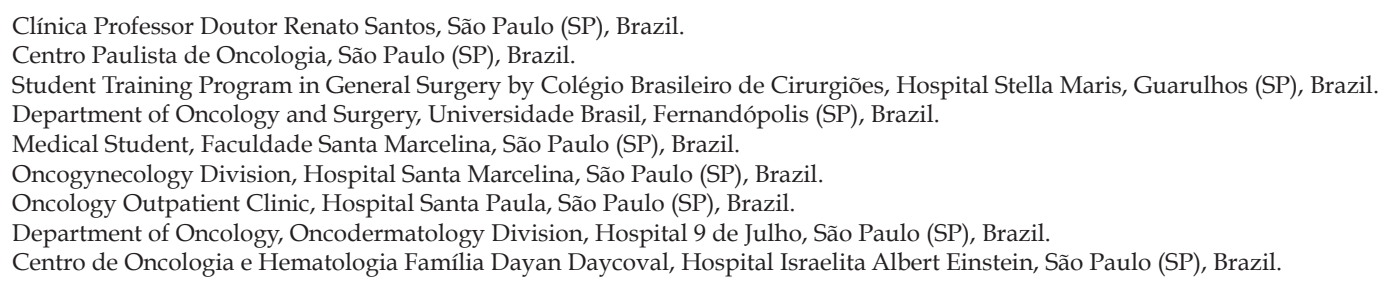


Currently, regression of the primary lesion is no longer considered a predictor of SLN involvement and is thus not used to indicate SLN biopsy. ${ }^{3,4}$

When conventional histopathology fails to identify SLN micrometastases, immunohistochemistry (IHC) is indicated, with the S100, HMB-45, and MART-1/Melan-A proteins as the target antigens. Molecular biology techniques such as RT-PCR (reverse transcription-polymerase chain reaction) and cell culture can identify even smaller amounts of metastatic cells in the sentinel lymph node, but they still lack clinical applicability in this scenario. SLN is the most important prognostic factor for patients with localized CM. ${ }^{5-8}$

SLN biopsy replaced elective lymphadenectomy in patients with clinically localized melanoma at high risk of lymph node metastasis. The current gold standard for patients with negative sentinel lymph node is clinical observation and monitoring of the nodal basin, and ultrasound can be considered in the follow-up.

\section{MANAGEMENT OF THE PATIENT WITH POSITIVE SENTINEL LYMPH NODE}

Traditionally, complete lymphadenectomy (CL) has been indicated in patients with positive SLN ${ }^{2}$ When lymphadenectomy is performed, the treatment goal is to remove other lymph nodes with metastases and theoretically interrupt spread of the melanoma to other organs. Metastases to non-sentinel lymph nodes have been observed in some $15-20 \%$ of specimens obtained from CL. ${ }^{9,10}$ The therapeutic effect of CL for patients without non-sentinel lymph node metastasis is unknown.

In addition to the possible therapeutic effect of complete lymphadenectomy, the approach stratifies nodal involvement as $\mathrm{N} 1$ (one lymph node involved), N2 (two to three lymph nodes involved), and N3 (four or more lymph nodes involved). The eighth edition of the AJCC Staging System adopts the nomenclature "a" (if detected by SLN biopsy), "b" (if detected clinically), or "c" (when in-transit or satellite lesions or microsatellite metastases are detected). ${ }^{11}$ Ten-year melanoma-specific survival differs considerably according to the nodal staging: $75 \%$ of N1 patients, $68 \%$ for N2, and only $47 \%$ for N3. ${ }^{12}$

The identification of risk factors for non-sentinel lymph node involvement among the patient's clinical characteristics and those of the primary lesion and sentinel lymph node can be used to select CM patients with SLN lymph node involvement that will not benefit from lymphadenectomy, thus preventing sequelae from the procedure.

Oncologists are currently debating whether or not to perform complete lymphadenectomy in CM patients with positive SLN biopsy, since recent randomized clinical trials results go against the traditionally recommended approach of complete lymph node dissection in the SLN basin.

\section{PRINCIPAL STUDIES AND RESULTS}

The Multicenter Selective Lymphadenectomy Trial (MSLT-I) is the only completed phase III randomized trial that has compared SLN biopsy versus no SLN biopsy in patients with localized CM and that assessed the role of complete lymphadenectomy. The study recruited 2,001 patients. In one arm of this study, patients were sub- mitted to broad excision of the melanoma with SLN biopsy. If the SLN was positive, patients underwent CL. If negative, they were simply observed. In the other arm, patients underwent broad excision alone and were followed. The results failed to show a difference between the two groups in either overall survival or melanoma-specific survival. However, in a post hoc analysis, for patients with Breslow thickness from $1.2 \mathrm{~mm}$ to $3.6 \mathrm{~mm}$ who had lymph node involvement (either at SLN biopsy or during follow-up), there was a benefit in melanoma-specific survival (MSS) for those submitted to immediate CL following a positive SLN result. Despite controversies concerning the therapeutic benefit of SLN biopsy, this study showed that SLN histopathological status was the most important prognostic factor for the survival of patients with localized $\mathrm{CM}$ (with clinically negative lymph nodes) and that SLN biopsy provides better disease-free survival (DFS).$^{13}$

Santos-Juanes et al. conducted a meta-analysis of six studies with 8,764 patients that underwent SLN biopsy and 11,054 that underwent only broad excision. Although four studies failed to observe a significant difference in survival, in the overall assessment SLN biopsy demonstrated superior evolution when compared to broad excision alone. ${ }^{14}$

The German study, Dermatologic Cooperative Oncology Group - Sentinel Lymph node Trial (DeCOG-SLT), was the first randomized clinical trial to assess the benefit of CL in melanoma patients with positive SLN biopsy. The trial enrolled 483 patients with cutaneous melanoma on the trunk and limbs, with a median follow-up of 35 months. The majority had micrometastases $<1.0 \mathrm{~mm}$ in the SLN (66\% of cases), and no difference was found in metastasis-free survival between the group with "dissected" nodal chain versus the group with spared nodal chain and followed with trimonthly ultrasound. The authors concluded that CL should not be performed in cases in which the SLN presents micrometastasis $\leq$ $1.0 \mathrm{~mm} \cdot{ }^{15}$ It should be noted that the authors reported difficulty in enrolling patients into a supposedly suboptimal therapy, and there was a clear decrease in statistical power from $80 \%$ to $50 \%$, which could not be resolved by increasing the follow-up.

In the wake of DeCOG-SLT, in the MSLT-II study, 1,934 patients with positive sentinel lymph node detected by histopathology or RT-PCR were randomized to receive immediate complete lymphadenectomy or watchful waiting with ultrasound. The primary outcome was melanoma-specific survival and the secondary outcomes were disease-free survival and non-sentinel lymph node involvement. The trial was negative for the primary outcome, essentially showing the same $86 \%$ three-year MSS in both study arms, even in the subgroup analysis. However, disease-free survival favored the lymphadenectomy arm by $5 \%$, due mainly to a $69 \%$ difference in regional lymph node disease-free survival, but with no change in distant disease-free survival. The authors concluded that complete lymphadenectomy following positive SLN biopsy can be waived, especially to spare patients from lymphedema (24\% in CL versus $6 \%$ in watchful waiting), since it had no impact on melanoma-specific survival, especially in patients with little nodal deposit in the SLN and who were willing to undergo rigorous ultrasound follow-up. Importantly, however, the study showed lower regional disease control and less precise prognostic stratification. ${ }^{16}$ 
EORTC 1208 MINITUB, a prospective trial still in progress, will assess whether the amount of tumor in the positive SLN is a valid predictor for sparing the patient from CL. Patients with intermediate Breslow thickness and minimal tumor volume, or SLN with minimal involvement (subcapsular $\leq 0.4 \mathrm{~mm}$, intraparenchymal $\leq$ $0.1 \mathrm{~mm}$ ), are followed to determine whether metastasis-free survival differs between those undergoing CL versus watchful waiting. It is not a randomized study, and patients are treated and followed according to each participating center and with ultrasound of the lymphatic basin draining the melanoma. Recruitment into this trial is scheduled for completion in 2019-2020. ${ }^{17}$

Burke et al. drew on concepts from theoretical biology to develop a Markov model to simulate the prognosis of hypothetical cohorts of patients with CM with SLN metastases, in two groups: those undergoing immediate lymphadenectomy and those followed and submitted to late lymphadenectomy in the presence of macroscopic nodal disease. The model assessed overall survival, life expectancy, and quality-adjusted life expectancy. Projected five-year overall survival for patients 50 years or older was $67.2 \%$, compared to $63.1 \%$ in the watchful waiting group, and this difference was statistically significant. The gain in life expectancy from immediate CL after positive SLN biopsy varied from 2.19 years for patients 30 to 70 years of age to 0.64 years for patients over 70 years. The gain in quality adjusted life expectancy from immediate CL varied from 1.39 years for patients 30 to 70 years of age to 0.36 years for those over 70 years. Sensitivity analysis was conducted to determine the results' stability in the Markov model, varying the parameters within the plausible variations according to the medical literature. For all the age cohorts, complete lymph node dissection was the best strategy when varying the risk of non-sentinel lymph node metastases from 15 to $30 \%$. For risk less than $7.8 \%$, watchful waiting showed the best result. ${ }^{18}$

\section{ACCURACY AND DISADVANTAGES OF SENTINEL LYMPH NODE BIOPSY}

The main disadvantage of SLN biopsy is the presence of false-negatives (FN). Assessment of false-negatives depends on the patient's evolution. False-negative SLN is defined as recurrence of the disease after negative SLN biopsy in the same lymph node basin. The FN rate is calculated by dividing the number of FNs by the sum of the true positives and false positives and varies from 8 to $20 \% .^{19-24}$ There are controversies concerning the overall survival of FN patients. In the MSLT-I study, overall survival of patients with false-negative SLN was similar to that of the watchful waiting group that developed lymph node metastasis but was significantly lower than in patients with positive SLN. Gambichler et al. found worse MSS in patients with false-negative SLN when compared to those with positive SLN. ${ }^{25}$

False-negative SLN can result from technical failure in the lymphoscintigraphy or in the surgical procedure. A contributing factor is massive SLN involvement altering the lymphatic drainage, resulting in failure to identify and remove the true SLN, as well as lack of intraoperative combined use of vital dye and gamma detection. Another cause lies in the histological protocol, purportedly missing the metastasis. Thus, one may either be examining a lymph node that is not the true SLN or missing occult micrometastases. Gershenwald et al. reassessed the histopathological examination of false-negative SLNs with serial slices of the lymph node and found occult metastases in five of seven patients who had nodal disease as the first site of recurrence. ${ }^{26}$ Finally, failure may be due to the presence of occult in-transit metastases that have still not reached the lymph node.

The addition of single photon emission computed tomography (SPECT-CT) to planar lymphoscintigraphy facilitates SLN identification and localization in CM, thereby decreasing the false-negative rate. ${ }^{27}$ Fluorescence, using hybrid tracers like indocyanine green and technetium-labelled nanocolloid ( $99 \mathrm{mTc})$, is a promising method for improving surgical precision in SLN biopsy. It promises to be highly useful for excising sentinel lymph nodes in difficult locations such as head and neck, mediastinum, and retroperitoneum. ${ }^{28}$ The addition of activated charcoal to vital dye increases the precision in the identification of the true SLN. ${ }^{29}$

False-positive SLN biopsies are also known to exist. This happens when immunohistochemistry (IHC) identifies niches of cells or single positive cells for melanoma markers, such as the above-mentioned MART-1 and S100, but these are not synonymous with CM metastases to the lymph node. As an example, up to $5.1 \%$ of patients with MART-1-positive cells can be false-positives as suggested by a study that found this incidence in SLN biopsies in patients without a history of melanoma. ${ }^{30}$ The most plausible explanations are the existence of nodal nevi and melanocytes that gained access to the lymphatics due to prior skin biopsy. ${ }^{31}$ To mitigate the FP rate, four accessory criteria can be used in the analysis of the SLN:

1- Existence of IHC-positive cells inside the lymph node: melanocytic cells in the nodal parenchyma are known to be malignant, while subcapsular or trabecular melanocytic cells are considered benign;

2- Cytologic characteristics, especially those related to the cell nucleus, such as nuclear pleomorphism, hyperchromasia, and enlargement, among others;

3- Absence or presence of evidence of proliferation, such as mitotic figures;

4- Positivity for HMB45 marker, which is less sensitive but highly specific for melanoma. ${ }^{32}$

Does a tool exist to increase prognostic accuracy in patients with positive SLN biopsy? The groundbreaking work of Hao et al., although not clinically applicable at present, identified two genes in the positive sentinel lymph node, namely PIGR, already correlated with early recurrence of other tumors, and TFAP2A, one of the genes responsible for acquisition of the malignant phenotype in melanoma. These two genes, together with the patient's clinical and pathological characteristics, were able to differentiate precisely between high-risk and low-risk groups for recurrence in the study cohort $($ AUC $=0.864) .{ }^{33}$

Positive non-sentinel lymph nodes in the presence of a positive sentinel lymph node

Nagaraja et al. conducted a meta-analysis to identify the predictive clinical and pathological variables for metastases in non-sentinel lymph nodes detected during complete lymphadenectomy in patients with positive SLN cutaneous melanoma. The results were analyzed by odds ratios with $95 \%$ confidence intervals in a random 
effects model. Fifty-four studies were analyzed, with a total of 8,388 patients, with incidence of metastases in non-sentinel lymph nodes varying from 8 to $38 \%$. The variables found in the primary lesion that presented significantly high odds of metastases (in a lymph node other than the sentinel lymph node) were presence of satellitosis, neurotropism, angiolymphatic invasion, and ulceration. In the sentinel lymph node, the variables extranodal extension, capsular involvement, extensive SLN involvement (Dewar), Starz 3, macrometastasis $>2 \mathrm{~mm}$, and more than one positive sentinel lymph node were statistically significant. Three characteristics were associated with absence of non-sentinel lymph node metastases: subcapsular localization, Rotterdam criterion $<0.1 \mathrm{~mm}$, and Starz I. ${ }^{34}$ The meta-analysis thus provides an additional argument for sparing $C L$ in patients with low metastatic load in the SLN, thereby preventing the morbidity associated with the procedure.

Just as research has endeavored to predict the involvement of non-sentinel lymph nodes after positive SLN biopsy, tools have also been created to predict the odds of sentinel lymph node involvement. In 2005, Wong et al. developed a nomogram based on age, primary site, Breslow thickness, Clark level, and ulceration, showing a negative predictive value of $90 \%$ with $0-3 \%$ error. Another model was developed by Mocellin et al., who reported a negative predictive value of $93 \%$, with $1-2 \%$ error. ${ }^{35}$ However, these models have not been validated in phase III trials and are not currently used in clinical practice.

Returning to the topic in the subtitle, there are various scores for the prediction of non-sentinel lymph node involvement, including the Rotterdam system, based on the measurement of the largest diameter of the largest metastatic deposit, dividing the positive SLNs in three groups: $<0.1 \mathrm{~mm} ; 0.1$ to $1.0 \mathrm{~mm}$; and $>1.0 \mathrm{~mm}$, predicting the risk of non-sentinel nodal involvement at $3 \%, 21 \%$, and $32 \%$, respectively. ${ }^{36}$ In the Dewar microanatomical classification of metastatic deposit in the SLN, the odds of additional lymph node involvement vary from $8 \%$ for subcapsular localization to $19 \%$ for parenchymal localization, reaching $40 \%$ in extensive involvement. There are other classifications, such as S, based on the depth of the metastatic deposit, dividing the positive SLNs into $<0.3 \mathrm{~mm}$ (S-I); $0.3 \mathrm{~mm}$ to $1.0 \mathrm{~mm}$ (SII); and $>1.0 \mathrm{~mm}$ (S-III); the Hannover-II classification, based on the largest dimension of the largest deposit, depth of the metastasis, and capsular involvement. ${ }^{37,38}$ The Non-Sentinel Node Risk Score (N-SNORE) considers gender, regression, proportion of involved SLN, maximum dimension, and perinodal lymphatic invasion. ${ }^{39}$ The EORTC melanoma groups adopts a Rotterdam-Dewar combination..$^{40}$ Sloan Kettering Memorial Cancer Center bases its approach on the size/ulceration score. ${ }^{41}$ The Rotterdam system is currently the most widely used. ${ }^{42}$

Based on these scores, when the patient is spared of $\mathrm{CL}$, lymph node basin ultrasound plays an important role in follow-up. The test allows diagnosing early recurrence, and fine needle aspiration biopsy (FNAB) is performed in the suspicious lymph nodes. However, the technique is operator-dependent, which requires the patient's availability for trimonthly ultrasound in the first year of follow-up, in addition to displaying some loss of sensitivity for lesions measuring less than $10 \mathrm{~mm} .{ }^{43}$

\section{CONCLUSION}

Currently, the change from a standard treatment to another potentially better one should be based on the best possible level of evidence and is worthy of note when there are phase III randomized clinical trials. Offering complete lymphadenectomy only to cutaneous melanoma patients with positive SLN that truly benefit from the procedure has been a highly desired step forward, since the complications from lymphadenectomy, especially lymphedema and cellulitis, significantly comprise the patient's quality of life. ${ }^{44}$

The vast majority of CMs send metastases initially to regional lymph nodes, and the sentinel lymph node is the principal marker for the condition. All stages of this technique need to be improved in order to decrease the false-negative and false-positive SLN rates. Progress is still needed in the stages that involve nuclear medicine, surgical technique, and anatomical pathology.

The results of the MSLT-I phase III randomized trial show that management based on sentinel lymph node biopsy in patients with melanoma of intermediate to high thickness leads to the best disease-free survival. Contrary to this reasoning, however, MSLT-II strongly suggests that complete lymphadenectomy following positive sentinel lymph node biopsy can be waived, since the overall survival and melanoma-specific survival rates were essentially the same.

Meanwhile, Burke et al., in a critical analysis using a Markov model, found that complete lymphadenectomy following positive SLN biopsy was associated with gains in overall survival and quality-adjusted life expectancy, compared to observation and late lymphadenectomy in patients with cutaneous melanoma that developed clinically apparent metastases.

EORTC 1208 - MINITUB will help answer this question. It is still not clear whether minimal SLN involvement (micrometastasis $<1 \mathrm{~mm}$ ) can harm prognosis in these patients, despite evidence to the contrary, nor whether it can be used as an exclusion criterion in clinical studies of adjuvant immunotherapy and target therapy with anti-BRAF associated with anti-MEK. ${ }^{45,46}$

Despite the false-negatives, false-positives, and lack of definition as to the value of SLN micrometastases, SLN biopsy is still the best methodology for staging initial melanomas. However, conducting complete lymph node dissection in all patients with positive SLN biopsy is not consistent with the current body of data in the literature, especially in patients with minimal SLN involvement. The procedure should be discussed in detail with the patient, listing the pros and cons of complete lymphadenectomy, considering the risk of regional recurrence, complications from the procedure, and the need to define the prognosis based on the result of the CL, and emphasizing the absence of benefit in overall survival from CL in prospective studies, despite the increase in lymph node recurrence-free survival. $\square$ 


\section{REFERENCES}

1. Weinstock MA. Epidemiology, etiology, and control of melanoma. Med Health $R$ I. $2001 ; 84: 234-6$

2. Coit D, Thompson J, Algazi A, Andtbacka R, Bichakjian C, Carson W, et al.Melanoma, Version 2.2016, NCCN Clinical Practice Guidelines in Oncology. JNCCN. Journal of the National Comprehensive Cancer Network. 2016:14:450-73.

3. Morris KT, Busam KJ, Bero S, Patel A, Brady MS. Primary cutaneous melanoma with regression does not require a lower threshold for sentinel lymph node biopsy. Ann Surg Oncol. 2008;15:316-22.

4. Socrier $\mathrm{Y}$, Lauwers-Cances V, Lamant L, Garrido I, Lauwers F, Lopez R, et al. Histological regression in primary melanoma: not a predictor of sentinel lymph node metastasis in a cohort of 397 patients. Br J Dermatol. 2010;162:830-4.

5. Morton D. Technical Details of Intraoperative Lymphatic Mapping for Early Stage Melanoma. Arch Surg. 1992 Apr;127:392-9.

6. Balch CM, Soong SJ, Gershenwald JE, Thompson JF, Reintgen DS, Cascinelli N, et al. Prognostic Factors Analysis of 17,600 Melanoma Patients: Validation of the American Joint Committee on Cancer Melanoma Staging System. J Clin Oncol. 2001:19:3622-34

7. Lee JH, Essner R, Torisu-Itakura H, Wanek L, Wang H, Morton DL. Factors Predictive of Tumor-Positive Nonsentinel Lymph Nodes After Tumor-Positive Sentinel Lymph Node Dissection for Melanoma. J Clin Oncol. 2004;22:3677-84.

8. Morton DL, Thompson JF, Cochran AJ, Mozzillo N, Elashoff R, Essner R, et al. Sentinel-Node Biopsy or Nodal Observation in Melanoma. N Engl J Med. 2006:355:1307-17.

9. Lee JH, Essner R, Torisu-ltakura H, Wanek L, Wang H, Morton DL. Factors Predictive of Tumor-Positive Nonsentinel Lymph Nodes After Tumor-Positive Sentinel Lymph Node Dissection for Melanoma. J Clin Oncol. 2004;22:3677-84.

10. Cascinelli N, Bombardieri E, Bufalino R, Camerini T, Carbone A, Clemente C, et al. Sentinel and Nonsentinel Node Status in Stage IB and II Melanoma Patients: TwoStep Prognostic Indicators of Survival. J Clin Oncol. 2006;24:4464-71.

11. Amin MB, Edge, Greene F, Byrd DR, Brookland RK, Washington MK, et al, eds. AJCC Cancer Staging Manual. 8th ed. New York: Springer International Publishing; 2017. p.563-585.

12. Balch CM, Gershenwald JE, Soong SJ, Thompson JF, Ding S, Byrd DR, et al. Multivariate Analysis of Prognostic Factors Among 2,313 Patients With Stage III Melanoma: Comparison of Nodal Micrometastases Versus Macrometastases. J Clin Oncol. 2010;28:2452-9

13. Morton DL, Thompson JF, Cochran AJ, Mozzillo N, Nieweg OE, Roses DF, et al. Final Trial Report of Sentinel-Node Biopsy versus Nodal Observation in Melanoma. N Engl J Med. 2014;370:599-609.

14. Santos-Juanes J, Fernández-Vega I, Galache Osuna C, Coto-Segura P, MartínezCamblor P. Sentinel lymph node biopsy plus wide local excision vs. wide location excision alone for primary cutaneous melanoma: a systematic review and metaanalysis. J Eur Acad Dermatol Venereol. 2017:31:241-6.

15. Leiter U, Stadler R, Mauch C, Hohenberger W, Brockmeyer N, Berking C, et al. Complete lymph node dissection versus no dissection in patients with sentinel lymph node biopsy positive melanoma (DeCOG-SLT): a multicentre, randomised, phase 3 trial. Lancet Oncol. 2016:17:757-67.

16. Faries MB, Thompson JF, Cochran AJ, Andtbacka RH, Mozzillo N, Zager JS, et al. Completion Dissection or Observation for Sentinel-Node Metastasis in Melanoma. N Engl J Med. 2017;376:2211-22.
17. Clinicaltrials.gov[Internet]. Minimal SN Tumor Burden (Minitub) [cited 2017 Dec 23]. Available from: https://clinicaltrials.gov/ct2/show/ NCT01942603?term $=01942603 \&$ rank $=1$

18. Burke EE, Portschy PR, Tuttle TM, Kuntz KM. Completion Lymph Node Dissection or Observation for Melanoma Sentinel Lymph Node Metastases: A Decision Analysis. Ann Surg Oncol. 2016;23:2772-8.

19. Nowecki Zl, Rutkowski P, Nasierowska-Guttmejer A, Ruka W. Survival analysis and clinicopathological factors associated with false-negative sentinel lymph node biopsy findings in patients with cutaneous melanoma. Ann Surg Oncol. 2006;13:1655-63.

20. Caracò C, Marone U, Celentano E, Botti G, Mozzillo N. Impact of false-negative sentinel lymph node biopsy on survival in patients with cutaneous melanoma. Ann Surg Oncol. 2007;14:2662-7.

21. Carlson GW, Page AJ, Cohen C, Parker D, Yaar R, Li A, et al. Regional recurrence after negative sentinel lymph node biopsy for melanoma. Ann Surg. 2008;248:378-86

22. Scoggins CR, Martin RC, Ross MI, Edwards MJ, Reintgen DS, Urist MM, et al. Factors Associated with False-Negative Sentinel Lymph Node Biopsy in Melanoma Patients. Ann Surg Oncol. 2010;17:709-17.

23. McDonald K, Page AJ, Jordan SW, Chu C, Hestley A, Delman KA, et al. Analysis of regional recurrence after negative sentinel lymph node biopsy for head and neck melanoma. Head Neck. 2013;35:667-71.

24. Lee DY, Huynh KT, Teng A, Lau BJ, Vitug S, Lee JH, et al. Predictors and Survival Impact of False-Negative Sentinel Nodes in Melanoma. Ann Surg Oncol. 2016;23:1012-8.

25. Gambichler T, Scholl L, Bechara FG, Stockfleth E, Stücker M. Worse outcome for patients with recurrent melanoma after negative sentinel lymph biopsy as compared to sentinel-positive patients. Eur J Surg Oncol. 2016;42:1420-6.

26. Gershenwald JE, Colome MI, Lee JE, Mansfield PF, Tseng C, Lee JJ, et al. Patterns of recurrence following a negative sentinel lymph node biopsy in 243 patients with stage I or II melanoma. J Clin Oncol. 1998;16:2253-60.

27. Chapman BC, Gleisner A, Kwak JJ, Hosokawa P, Paniccia A, Merkow JS, et al SPECT/CT Improves Detection of Metastatic Sentinel Lymph Nodes in Patients with Head and Neck Melanoma. Ann Surg Oncol. 2016;23:2652-7.

28. KleinJan GH, Bunschoten A, van den Berg NS, Olmos RA, Klop WM, Horenblas S, et al. Fluorescence guided surgery and tracer-dose, fact or fiction? Eur J Nucl Med Mol Imaging. 2016;43:1857-67.

29. Pereira R, Oliveira Filho R, Zuliani C, Vieira R, Enokihara M, Pinheiro L. Vital dye and carbon solution for sentinel lymph node biopsy in rats. Einstein. 2008;6:463-6.Yan $\mathrm{S}$, Brennick JB. False-positive rate of the immunoperoxidase stains for MART1/ MelanA in lymph nodes. Am J Surg Pathol. 2004;28:596-600.

30. Bautista NC, Cohen S, Anders KH. Benign melanocytic nevus cells in axillary lymph nodes. A prospective incidence and immunohistochemical study with literature review. Am J Clin Pathol. 1994;102:102-8.

31. Brennick JB, Yan S. False-positive cells in sentinel lymph nodes. Semin Diagn Pathol. 2008;25:116-9.

32. Hao H, Xiao D, Pan J, Qu J, Egger M, Waigel S, Sanders MA, et al. Sentinel Lymph Node Genes to Predict Prognosis in Node-Positive Melanoma Patients. Ann Surg Oncol. 2017:24:108-116. 
33. Nagaraja V, Eslick GD. Is complete lymph node dissection after a positive sentinel lymph node biopsy for cutaneous melanoma always necessary? A meta-analysis. Eur J Surg Oncol. 2013;39:669-80.

34. Mocellin S, Thompson JF, Pasquali S, Montesco MC, Pilati P, Nitti D, et al. Sentinel Node Status Prediction by Four Statistical Models. Ann Surg. 2009;250:964-9.

35. van Akkooi AC, de Wilt JH, Verhoef C, Schmitz Pl, van Geel AN, Eggermont AM, et al. Clinical relevance of melanoma micrometastases $(<0.1 \mathrm{~mm})$ in sentinel nodes: are these nodes to be considered negative? Ann Oncol. 2006;17:1578-85.

36. Starz H, Siedlecki K, Balda BR. Sentinel lymphonodectomy and s-classification: a successful strategy for better prediction and improvement of outcome of melanoma. Ann Surg Oncol. 2004:11:162S-8S.

37. Meier A, Satzger I, Völker B, Kapp A, Gutzmer R. Comparison of classification systems in melanoma sentinel lymph nodes-An analysis of 697 patients from a single center. Cancer. 2010;116:3178-88.

38. Murali R, Desilva C, Thompson JF, Scolyer RA. Non-Sentinel Node Risk Score (N-SNORE): a scoring system for accurately stratifying risk of non-sentinel node positivity in patients with cutaneous melanoma with positive sentinel lymph nodes. J Clin Oncol. 2010 0ct 10;28:4441-9.
39. van der Ploeg AP, van Akkooi AC, Rutkowski P, Nowecki Zl, Michej W, Mitra A, et al. Prognosis in patients with sentinel node-positive melanoma is accurately defined by the combined Rotterdam tumor load and Dewar topography criteria. J Clin Oncol. 2011;29:2206-14.

40. Reeves ME, Delgado R, Busam KJ, Brady MS, Coit DG. Prediction of Nonsentinel Lymph Node Status in Melanoma. Ann Surg Oncol. 2003;10:27-31.

41. van Akkooi AC, Nowecki Zl, Voit C, Schäfer-Hesterberg G, Michej W, de Wilt JH, et al. Sentinel Node Tumor Burden According to the Rotterdam Criteria Is the Most Important Prognostic Factor for Survival in Melanoma Patients. Ann Surg. 2008;248:949-55

42. Voit C, Mayer T, Proebstle TM, Weber L, Kron M, Krupienski M, et al. Ultrasoundguided fine-needle aspiration cytology in the early detection of melanoma metastases. Cancer. 2000;90:186-93.

43. Liu JB, Bilimoria KY. Weighing the value of completion nodal dissection for melanoma. J Surg Oncol. 2016;114:281-7.

44. Eggermont AM, Chiarion-Sileni V, Grob JJ, Dummer R, Wolchok JD, Schmidt H, et al. Adjuvant ipilimumab versus placebo after complete resection of high-risk stage III melanoma (EORTC 18071): a randomised, double-blind, phase 3 trial. Lancet Oncol. 2015;16:522-30.

45. Long GV, Hauschild A, Santinami M, Atkinson V, Mandalà M, Chiarion-Sileni V, et al. Adjuvant Dabrafenib plus Trametinib in Stage III BRAF-Mutated Melanoma. N Engl J Med. 2017;377:1813-23.

\section{AUTHORS CONTRIBUTION}

\section{Daniel Eiger}

(iD) ORCID 0000-0002-9879-6048

Approval of the final version of the manuscript; Elaboration and writing of the manuscript; Obtaining, analyzing and interpreting the data; Critical review of the literature; Critical review of the manuscript

Daniel Arcuschin de Oliveira

iD ORCID 0000-0003-0591-041X

Approval of the final version of the manuscript; Conception and planning if the study; Obtaining, analyzing and interpreting the data

Renato Leão de Oliveira

D ORCID 0000-0002-6769-2414

\section{Murilo Costa Sousa \\ ORCID 0000-0002-0542-3200 \\ Elaboration and writing of the manuscript \\ Mireille Darc Cavalcante Brandão \\ ORCID 0000-0003-4676-157X}

Approval of the final version of the manuscript; Conception and planning if the study; Critical review of the literature

Renato Santos de Oliveira Filho

(iD) ORCID 0000-0002-7464-973X

Approval of the final version of the manuscript; Elaboration and writing of the manuscript; Obtaining, analyzing and interpreting the data; Critical review of the literature Critical review of the manuscript

How to cite this article: Eiger D, Oliveira DA, Oliveira RL, Sousa MC, Brandão MDC, Oliveira Filho RS. Complete lymphadenectomy following positive sentinel lymph node biopsy in cutaneous melanoma: a critical review. An Bras Dermatol. 2018;93(4):553-8. 